\title{
Best Practices for Implementing Electronic Disease Surveillance Systems in Resources-constrained Settings
}

\author{
Carmen C. Mundaca*1, Vivek Singh ${ }^{2}$, Kayumba Kizito ${ }^{3}$ and Julie Pavlin ${ }^{4}$ \\ 'Uniformed Services University, Bethesda, MD, USA; ${ }^{2}$ Indian Institute of Public Health, Hyderabed, India; ${ }^{3}$ Voxiva SARL, Kigali, \\ Rwanda; ${ }^{4}$ Armed Forces Health Surveillance Center, Silver Spring, MD, USA
}

\section{Objective}

The panel will present successful and unsuccessful practices, challenges and lessons learned when implementing electronic surveillance tools in the developing world, and a best practices model that aims to guide and facilitate this process. Panelists and audience will discuss the validity of this model, and identify potential countries where it could be tested.

\section{Introduction}

Developing countries bear the highest burden of infectious diseases, and therefore play a key role in the detection of emerging disease threats. The ability of these countries to detect such events allows an adequate response preventing its spread and reducing the morbidity and mortality of communities across the world [1].

Due to the importance of strengthening the surveillance capabilities of developing countries, resources need to be dedicated to this effort. International organizations often provide technical and financial support when countries need assistance. When implementing such systems, the countries and supporting organizations face challenges not limited to scarce resources. These countries often also face political and social instability, and cultural and religious practices that may put their populations at greater risk of these events [2].

In addition, the disease surveillance field lacks clear guidance on how to initiate or improve the implementation process for these systems. The experiences described in the literature are often presented as partial evaluations using the US Centers for Disease Control and Prevention guidelines, without providing an explanation of the key factors that are related to successful or unsuccessful experiences in such settings [3]. Public health practitioners at the ministries of health $(\mathrm{MOH})$ and international organizations would greatly benefit from a model that considers these factors to serve as a guideline for a positive implementation outcome and an effective use of resources.

\section{Methods}

Three panelists will present a 15 minute talk each. The first two panelists will describe their experiences, with a special focus on the challenges encountered and the lessons learned when implementing electronic tools for disease surveillance. The first disease surveillance project was implemented using mobile phone technology in the state of Andhra Pradesh in India. The second experience presented will be based on the implementation of a national phone-based and webbased tool for communicable diseases in Rwanda.

The third panelist will present a best practices model developed with in-country data from the $\mathrm{MOH}$ and Armed Forces in Peru, and triangulated with findings in the literature and an expert consensus workshop organized in Silver Spring, MD in 2012. This model will inform the audience on the factors related to successful implementation experiences at three levels of the system: 1) at the high administrative level, 2) at the national and regional surveillance team level, and 3) surveillance site level.

\section{Results}

The first 15 minutes of discussion will be allocated to questions from the audience to the first two panelists on the presenters' implementation experiences. The moderator will also encourage the audience during this time to share their own experiences. The remainder of the session will focus on discussing the model presented by the third panelist. The moderator will ask the two first panelists for agreements, and disagreements on the model presented, and then will ask participants to comment on the model based on their experience. The participants will also share ideas on potential countries where this model could be tested for validation or refinement.

\section{Keywords}

electronic tools; implementation process; developing countries

\section{References}

1. Sturtevant, J.L., A. Anema, and J.S. Brownstein, The new International Health Regulations: considerations for global public health surveillance. Disaster Med Public Health Prep, 2007. 1(2): p. 117-21.

2. Calain, P., From the field side of the binoculars: a different view on global public health surveillance. Health Policy Plan, 2007. 22(1): p. $13-20$.

3. CDC, Updated Guidelines for Evaluating Public Health Surveillance System, 2001, Centers for Disease Control and Prevention.

*Carmen C. Mundaca

E-mail: mundaca.cecilia@gmail.com 